\section{Bessere Atmung nach Thermoplastie}

\author{
Vor allem bei Patienten mit schwerem Asthma bronchiale ist die \\ Masse der glatten Atemwegsmuskulatur stark vermehrt. Das neue \\ Verfahren der bronchialen Thermoplastie reduziert die glatte \\ Muskulatur und vermindert damit die Bronchokonstriktion.
}

l: $\mathrm{n}$ einer kontrollierten Studie wurden an 112 Patienten mit moderatem bis schwerem Asthma Wirksamkeit und Verträglichkeit der bronchialen Thermoplastie untersucht. Bei dieser Methode werden die Atemwege mit einem Durchmesser zwischen 3 und $10 \mathrm{~mm}$ per Bronchoskop kontrolliert erhitzt. Die Patienten wurden randomisiert einer Interventionsgruppe oder einer Kontrollgruppe zugewiesen und dann über ein Jahr beobachtet. Primäres Studienziel war die Häufigkeit von milden Exazerbationen innerhalb von drei zweiwöchigen Phasen ohne Anwendung von langwirksamen $\beta_{2}$-Agonisten (LABA). Die Intervention bestand in dreimaliger Durchführung einer bronchialen Thermoplastie.

In der Interventionsgruppe reduzierte sich im Vergleich zum Basiswert die
Häufigkeit milder Exazerbationen, während sie in der Kontrollgruppe unverändert blieb $(p=0,005)$. Nach zwölf Monaten ergaben sich außerdem bei den Patienten nach bronchialer Thermoplastie signifikante Verbesserungen beim morgendlichen exspiratorischen Peak-Flow, bei den Bewertungen im Asthma Quality of Life Questionnaire (AQLQ) und beim Asthma Control Questionnaire (ACQ), beim Prozentsatz symptomfreier Tage und bei den Symptomscores. Außerdem wurden in den zweiwöchigen LABA-Abstinenzphasen weniger zusätzliche Bedarfsmedikamente benötigt.

Unmittelbar nach der bronchialen Thermoplastie traten häufiger unerwünschte Effekte auf als ohne Intervention. Nach Abschluss der Behandlung bestand aber kein Unterschied mehr zwischen den beiden Gruppen.

\title{
Kein Unterschied bei den Killer-T-Zellen
}

Die Entzündungsreaktion beim allergischen Asthma wird durch $\mathrm{TH}_{2}$ -

Zellen vermittelt, die Antigenerkennung erfolgt Klasse-II-MHCrestringiert. Sind diese Zellen den erst kürzlich entdeckten CD1drestringierten invarianten natürlichen Killer-T-Zellen zuzuordnen?

D ie invarianten natürlichen KillerT-Zellen stellen eine neu entdeckte Subgruppe von immunregulatorischen T-Lymphozyten dar, bei denen die Antigen-Erkennung nicht über MHC-II-assozierte Komplexe, sondern über das CD1d-Molekül erfolgt. In drei kürzlich erschienenen Publikationen wird über erhöhte Zahlen dieser Zellen in den Atemwegen von Asthmapatienten berichtet. Dabei gibt es allerdings unterschiedliche Befunde.

Britische Forscher untersuchten, inwieweit die Zahl der invarianten natürlichen Killer-T-Zellen mit der Schwere der Erkrankung variiert und ob eine Erhöhung der Zellzahl auch bei Pa- tienten mit einer chronischen obstruktiven Lungenerkrankung (COPD) zu finden ist. Dazu gewannen sie aus bronchoalveolärer Lavage, induziertem Sputum und Bronchialbiopsien Proben von Patienten mit leichtem bis mittelschwerem Asthma, von Patienten mit COPD sowie von gesunden Kontrollpersonen und suchten darin nach invarianten natürlichen Killer-T-Zellen. Zum Einsatz kam dabei eine Kombination von Durchflusszytometrie mit verschiedenen spezifischen monoklonalen Antikörpern und Polymerasekettenreaktion, welche die Genexpression der für diese Zellpopulation typischen V $\alpha 24-$ und V $\beta 11$ Domänen detektieren sollte.

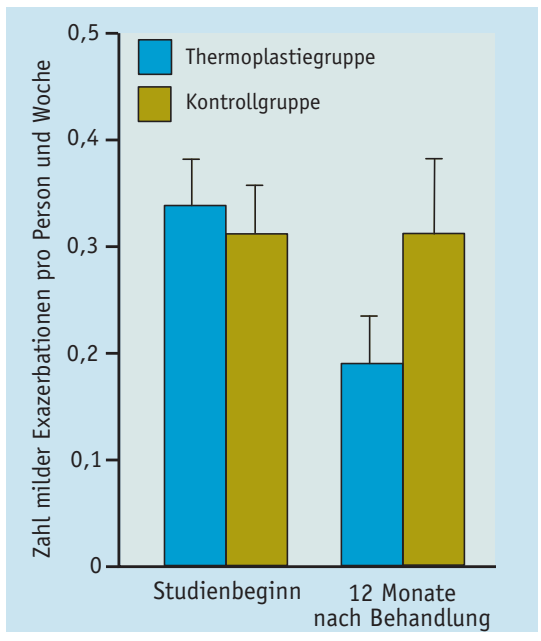

Eine bronchiale Thermoplastie reduziert die Anzahl milder Exazerbationen im Vergleich zur Kontrollgruppe.

Fazit: Die bronchiale Thermoplastie führt bei Patienten mit moderatem bis schwerem Asthma zu einer Verbesserung der Krankheitskontrolle.

$b k$

Cox $\mathrm{G}$ et al. Asthma control during the year after bronchial thermoplasty. $\mathrm{N}$ Engl J Med 2007; 356: 1327-37
Bei allen Patientengruppen fanden sich in diesem Versuchsansatz weniger als 2\% invariante natürliche Killer-TZellen. Die Diskrepanz zu den Ergebnissen vorheriger Studien erklären die Autoren unter anderem mit einer ungenügenden Aufbereitung der Proben. Außerdem können Makrophagen und Zelltrümmer durch Autofluoreszenz ebenfalls die Messergebnisse der Durchflusszytometrie beeinflussen.

Fazit: Invariante natürliche Killer-TZellen finden sich nur in kleiner Zahl in den Atemwegen von Patienten mit Asthma oder COPD sowie von Gesunden. Daher sollten auch weiterhin primär Therapiestrategien verfolgt werden, die auf Klasse-II-MHC-restringierte T-Zellen zielen.

Vijayanand P et al. Invariant natural killer $T$ cells in asthma and chronic obstructive pulmonary disease. N Engl J Med 2007; 356: 1410-22 